\title{
Perceptions and Positionings of Colleges in New York City: A Longitudinal Study of Brand Images
}

Received (in revised form): October 21, 2004

\section{Sylvia D. Clark}

Dr. Sylvia D. Clark is an associate professor of marketing at St. John's University in Staten Island, New York. She has taught undergraduate classes in consumer behavior, marketing research, and retailing, as well as graduate courses in international marketing and an MBA marketing seminar. Her research interests include brand management and measures of market orientation.

\begin{abstract}
A study of high school seniors' perceptions of colleges, conducted in 1979, was replicated 23 years later. The study affords an opportunity to examine perceptual changes over time and provides an additional perspective on the positioning of colleges, apart from other well-known surveys. This information may be useful for those involved in marketing and developing images for the schools included in the study.
\end{abstract}

\section{Keywords:}

brand names, brand image, brand differentiation, corporate image, education policy, universities and colleges

\section{Introduction}

Meet "Nessie." NSSE, or the National Survey of Student Engagement, is the latest resource available for comparing

Author's Contact Address:

Sylvia D. Clark

Associate Professor of Marketing

Peter J. Tobin College of Business

St. John's University

300 Howard Avenue

Staten Island, NY 10301, USA

Phone: + 17183904552

Fax: + 17188168602

Email: clark1094@aol.com colleges and universities. Launched by Indiana University in 1999, NSSE is viewed as a means of countering the US Newes rankings. The survey questions more than 155,000 students at 470 colleges, covering areas not touched upon by the traditional rankings. Included are such measures as time spent in preparing for class, typical amount read, and frequency of collaboration on faculty research.

NSSE joins a growing arsenal of information employable by colleges in their quest for students. In a crowded marketplace, colleges compete for new students on a number of different dimensions, ranging from cost to extracurricular activities. While wellpublicized rankings provide a convenient benchmark, they are merely a starting point, covering a selection of measures for a subgroup of top schools.

The present research offers an alternate perspective, by assessing high school seniors' perceptions of colleges. It also affords an opportunity to examine perceptual changes over time. The initial study, conducted in 1979, was replicated 23 years later. Such information may be very useful for those involved in marketing and developing images for their schools. 
The paper begins with some background information on college ranking measures, then describe our alternative methodology, the results of the 1979 and 2002 studies, and conclude with some recommendations for education marketers.

\section{Background}

Colleges have become increasingly competitive, particularly over the past 20 years. As the baby boomers aged out of their college years, they were supplanted by a substantially smaller generation. At the same time, US Neres \& World Report fed the frenzy, introducing its nowfamous system of rankings in 1983.

The US Neres \& World Report rankings set the standard for the onslaught of data to follow. The measures are produced by combining expert opinion with a variety of statistical indicators, yielding rankings of programs across several popular areas of study. ${ }^{1}$ The most recent survey (2002) gathered data from more than 1,000 programs and 9,000 academic and other professionals. ${ }^{2}$ Findings are available on a website and also published in a guidebook.

On the heels of the US Neres data came the biennial Business Week survey, introduced in 1988. The Business Week data offered a different slant, basing the rankings on surveys of alumni and recruiters. The most recent wave, conducted in 2000, included the views of more than 10,000 alumni and nearly 250 companies. ${ }^{3}$

Over the years, other evaluative measures have been developed, including Time's "best college" series and the aforementioned NSSE. But where have all these measures led? In the matter of "rank injustice," Cronin questions the value of what he considers measurement mania. ${ }^{4}$ He points to flaws in the methodology (e.g., questionnaire design, sampling) which could surely compromise the validity of the findings. He also notes the absurdity of using rankings for an extremely small number of programs and pokes fun at the esoteric bases for evaluation (e.g., "wiredness" to denote technological capability).

One recent study went beyond the rankings in an effort to understand structural differences across programs. Segev and associates noted that universities have been forced to continually reevaluate their curricula, partly because of the pressure engendered by the widespread use of rankings. ${ }^{5}$ With the US Neres $\&$ World Report data as their springboard, the researchers studied the top 25 business schools. MBA programs were compared based on content alone (i.e., the mix of core courses and concentration areas offered), purposefully excluding other factors, such as institutional reputation and quality of faculty. Coplot analysis yielded six clusters of schools, with each of the five "best" programs falling into a separate cluster. Hence there appeared to be no single best program structure: content itself was neither a distinguishing feature nor a source of superior performance.

Segev's findings suggest that, as with many common consumer products and services, subjective factors may play a more important role in the potential student's decision. Lowry and Owens explore this very notion, recommending that positioning strategy for a college be viewed as similar to that required for a typical product. ${ }^{6}$ While the ever-popular rankings may be used as a secondary source, the authors suggest conducting primary research to discern the school's present image. The traditional audience of prospective students-high school juniors and seniors-is the prime target, but views 
of other significant publics (e.g., parents, alumni, faculty) may also be sought in focus groups or surveys. A wide variety of potential positionings may be utilized as draws, among them cost, size, location, and even beauty of the campus.

In a slightly different vein, Hon and Brunner propose that the emotional appeal of an organization has a major role in building public perception. ${ }^{7}$ They surveyed more than 400 students currently enrolled at University of Florida to examine various facets of their attachment to the school. Measures of trust and satisfaction were found to be most descriptive of students' relationships with the school. The researchers point to the parallel to a typical customer exchange. Once again, perceptual factors may serve as a key means of differentiating colleges.

\section{Methodology}

The initial study was conducted in 1979 and replicated in 2002. Sixteen four-year colleges in New York City were evaluated by college-bound high school seniors from the same area. These colleges comprised nine private institutions: St. John's University, Long Island University (LIU), Pace University, New York University, Wagner College, St. Francis College, Fordham University, Columbia University, and Polytechnic University (Polytech), and seven public institutions all part of the City University of New York: City College, Baruch College, Brooklyn College, Queens College, John Jay College of Criminal Justice, College of Staten Island (CSI), and Hunter College. A questionnaire was used to obtain information about the 16 colleges on eight attributes measured on seven-point semantic differential scales: accessibility by public transportation, extent of campus facilities, cost, academic reputation, number of courses, specialization of educational offerings, size of student body, and extracurricular activities (see Appendix). These data yielded a simplespace configuration of the 16 stimuli, based on the dissimilarities among them as perceived by the respondents.

\section{Sample description}

For the initial 1979 study, the sample size was 60 , while in 2002, 138 usable questionnaires were collected. In both studies, respondents came from a variety of high schools throughout the New York City metropolitan area, including public, private, same-sex, and specialized mathand science-oriented schools. Both waves (1979 and 2002) used comparable selfadministered forms supervised by college undergraduates who collected the data for class credit. These undergraduates enlisted their alma maters and younger relatives and friends who were still in high school to take part in the research. While this was a convenience sample, for the purposes of this study, the sample was optimal since the respondents were highly involved in the college search process.

\section{Results}

In both waves (1979 and 2002), the respondents were college-bound high school seniors from New York City. Specifically, the 1979 sample was 37 percent male and 63 percent female. The vast majority $(88 \%)$ of these students reported having a high school average of 80 or above and most $(73 \%)$ anticipated working part-time while attending college. The 2002 sample was very similar, consisting of 39 percent males and 61 percent females, with 83 percent selfreporting high school averages of 80 or above. However, when it comes to combining work and college, the two groups differ in that more of the 2002 students intend to work. Compared with 


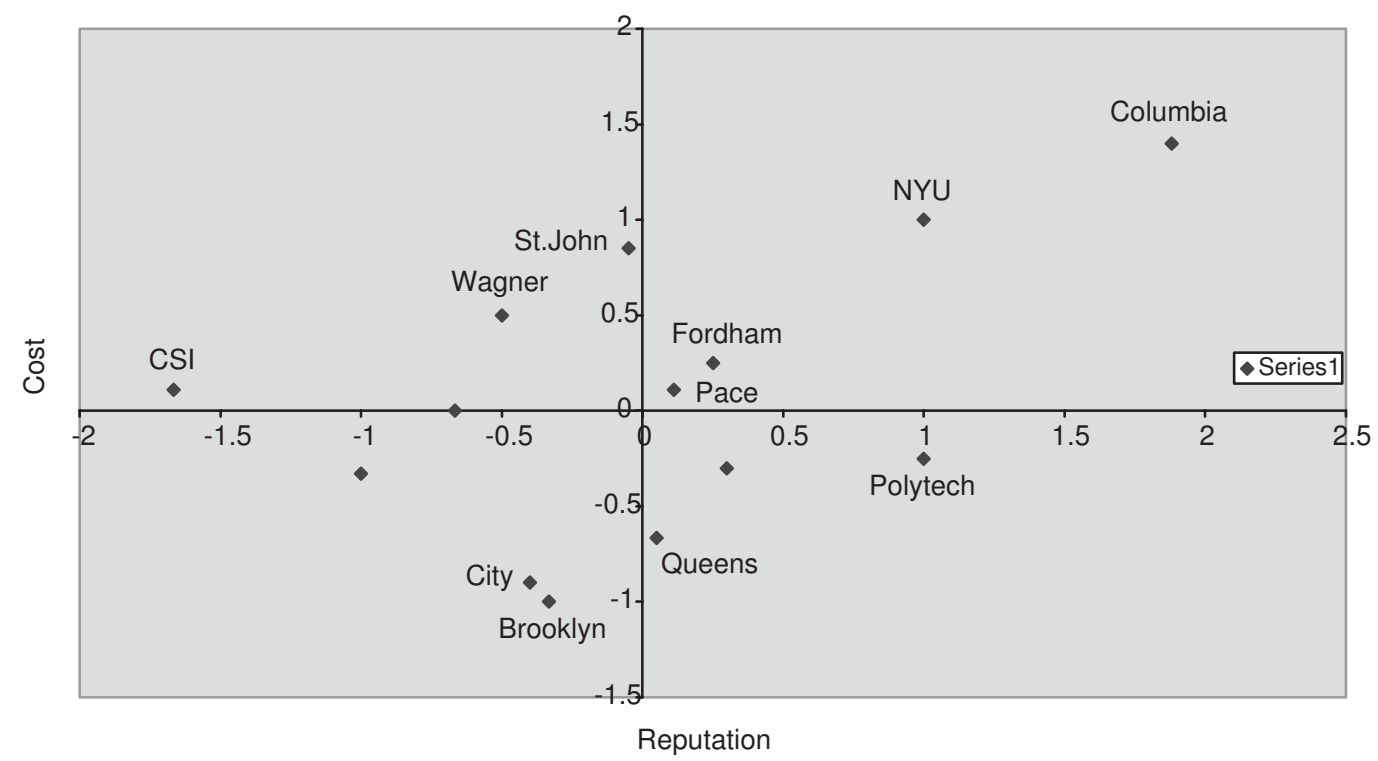

Figure 1: Derived stimulus configuration-Euclidean distance model (1979)

the 1979 group in which no respondents indicated that they would work full-time while in college, 6 percent of the 2002 sample expressed this intention with another 71 percent stating that they would work part-time.

Multidimensional scaling was used to form a perceptual map of similarities among the colleges. The 1979 data reveal a large cluster of city schools, that is, public City University colleges, in the two lower quadrants (Figure 1). One interpretation is that the horizontal axis represents a "reputation continuum," from low-quality to high-quality, while the vertical axis functions as a "cost continuum," from high cost to low cost. Respondents obviously had some knowledge of the substantially lower cost of attending a city school. Additionally, all the city schools, with the exception of Hunter and Queens colleges, are on the negative side of the continuum in terms of academic reputation as well. The open admissions policy of that time period could possibly be a contributing factor to this negative image.

The perceptual map clearly illustrates that Columbia was well ahead of the other colleges in 1979 in terms of academic reputation while CSI was perceived as greatly inferior to other choices. In terms of the cost differential, Columbia and Brooklyn College were perceived as being least similar. However, any city school could have been in Brooklyn College's place as they all charged the same low tuition.

The 2002 data produced a different view of respondents' perceptions. As indicated in Figure 2, the vertical axis represents a "reputation continuum," from low quality to high quality. The horizontal axis was more difficult to interpret. As in the 1979 study, Columbia clearly stands out for its superior reputation. However, it is now slightly surpassed by NYU, which appears to have made a significant gain in reputation over the past 22 years. 


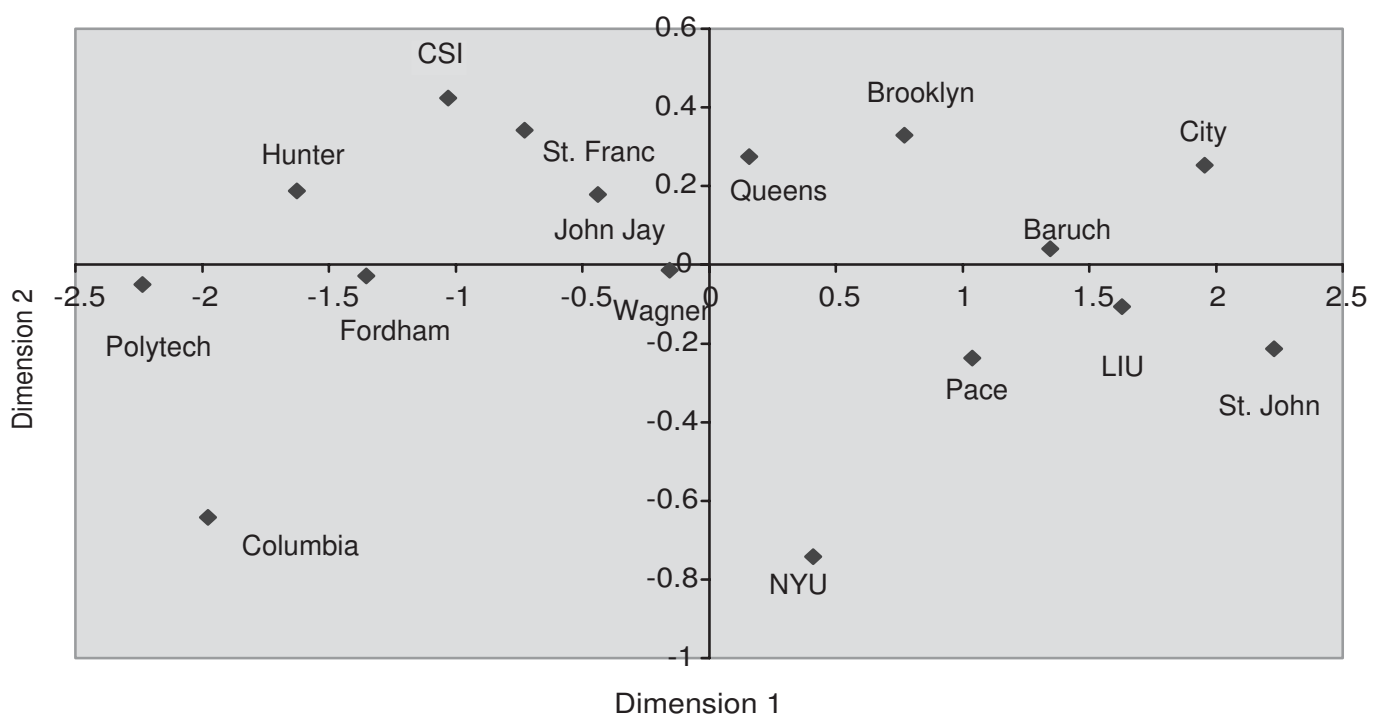

Figure 2: Derived stimulus configuration-Euclidean distance model (2002)

In order to further develop profiles of the colleges, the average scores across the years for each of the 16 colleges on each attribute was also examined. Examination of the averages of each of the eight attributes in both 1979 and 2002 revealed particular areas in which schools were deemed to either excel or fall short (see Tables 1 and 2). Since these attributes were measured on seven-point scales, the median value should be four. By looking for averages with deviations of 10 percent or more (i.e., 0.7 or greater) above or below the midpoint, one can observe which colleges stand out in respondents' minds on various attributes (see Tables 3 and 4). The differences in scores between 1979 and 2002 are presented in Table 5. A snapshot summary (see Table 6) of the significant differences for each of these years clearly shows that Columbia and NYU have consistently had the most distinct positionings throughout the years, with scores significantly different from the mid-point on most of the attributes.
While these two colleges are perceived as expensive, they also are noted for having extensive campus facilities, course offerings and extracurricular activities, favorable academic reputations, and large student bodies. Interestingly, the 2002 study indicates that NYU has surpassed Columbia in academic reputation (2.51 vs. 2.71). Additionally, NYU is now perceived as offering a specialized education while Columbia has lost this distinction. Also noteworthy is the perception of Baruch as now offering a specialized education.

These findings regarding specialized education are consistent with the US Neres ๙ World Report College Rankings (2003). Both NYU and Baruch are listed under "best undergraduate business programs" while Columbia is categorized under "best engineering programs.” However, several other colleges, including St. John's, City College, Pace, Fordham, and Polytech, were also noted in the US Neres \& World Report survey for "best programs" in business or engineering although our 
Table 1: Average rating on college attributes-1979

\begin{tabular}{|c|c|c|c|c|c|c|c|c|}
\hline & $\begin{array}{l}\text { Transportation } \\
1=\text { easy, } \\
7=\text { hard }\end{array}$ & $\begin{array}{l}\text { Campus } \\
\text { facilities } \\
\text { 1=extensive, } \\
7=\text { limited }\end{array}$ & $\begin{array}{l}\text { Cost } \\
\text { 1=high, } \\
7=\text { low }\end{array}$ & $\begin{array}{l}\text { Reputation } \\
\text { 1=high, } \\
\text { 7=low }\end{array}$ & $\begin{array}{l}\begin{array}{l}\text { Course } \\
\text { offerings }\end{array} \\
\text { 1=limited, } \\
7=\text { extensive }\end{array}$ & $\begin{array}{l}\text { Educ. } \\
\text { type } \\
1=\text { special, } \\
7=\text { genl. }\end{array}$ & $\begin{array}{l}\text { Student } \\
\text { body } \\
1=\text { small, } \\
7=\text { large }\end{array}$ & $\begin{array}{l}\text { Extracurricular } \\
\text { activities } \\
\text { 1=wide, } \\
7=\text { narrow } \\
\text { selection }\end{array}$ \\
\hline St. John & 3.75 & 3.33 & 3.43 & 3.07 & 5.02 & 3.87 & 4.50 & 2.87 \\
\hline City & 3.53 & 4.27 & 5.85 & 4.57 & 4.73 & 4.77 & 5.50 & 3.85 \\
\hline LIU & 4.67 & 3.98 & 4.00 & 4.15 & 4.80 & 4.27 & 4.63 & 3.68 \\
\hline Baruch & 3.68 & 4.22 & 5.37 & 3.67 & 4.37 & 3.50 & 4.77 & 4.08 \\
\hline Pace & 3.32 & 4.03 & 3.95 & 3.45 & 4.42 & 3.70 & 4.77 & 3.80 \\
\hline Bklyn & 3.05 & 3.95 & 5.72 & 4.37 & 4.57 & 4.98 & 5.42 & 3.82 \\
\hline NYU & 3.17 & 2.97 & 2.62 & 2.45 & 5.65 & 3.50 & 5.77 & 3.12 \\
\hline Queens & 4.28 & 3.70 & 5.45 & 3.75 & 4.60 & 4.67 & 5.37 & 3.65 \\
\hline Wagner & 4.53 & 3.25 & 3.15 & 3.75 & 4.65 & 4.23 & 3.93 & 3.32 \\
\hline JohnJay & 4.05 & 4.73 & 5.18 & 4.07 & 3.66 & 3.08 & 3.77 & 4.38 \\
\hline St.Franc & 3.97 & 4.13 & 4.02 & 3.88 & 4.18 & 4.67 & 3.97 & 3.43 \\
\hline CSI & 4.56 & 3.70 & 5.37 & 4.92 & 3.78 & 4.77 & 4.08 & 4.20 \\
\hline Fordham & 4.25 & 3.48 & 3.52 & 2.92 & 5.00 & 3.62 & 4.60 & 3.73 \\
\hline Hunter & 3.72 & 3.78 & 5.03 & 3.02 & 4.47 & 3.85 & 4.97 & 3.97 \\
\hline Columbia & 3.82 & 2.72 & 2.10 & 1.72 & 5.75 & 3.18 & 5.32 & 3.28 \\
\hline Polytech & 4.20 & 3.83 & 3.37 & 2.67 & 4.37 & 2.30 & 4.00 & 4.20 \\
\hline
\end{tabular}

Table 2: Average rating on college attributes-2002

\begin{tabular}{|c|c|c|c|c|c|c|c|c|}
\hline & $\begin{array}{l}\text { Transportation } \\
1=\text { easy, } \\
7=\text { hard }\end{array}$ & $\begin{array}{l}\text { Campus } \\
\text { facilities } \\
1=\text { extensive, } \\
7=\text { limited }\end{array}$ & $\begin{array}{l}\text { Cost } \\
\text { 1=high, } \\
7=\text { low }\end{array}$ & $\begin{array}{l}\text { Reputation } \\
\text { 1=high, } \\
7=\text { low }\end{array}$ & $\begin{array}{l}\begin{array}{l}\text { Course } \\
\text { offerings }\end{array} \\
1=\text { limited, } \\
7=\text { extensive }\end{array}$ & $\begin{array}{l}\text { Educ. } \\
\text { type } \\
1=\text { special, } \\
7=\text { genl. }\end{array}$ & $\begin{array}{l}\text { Student } \\
\text { body } \\
1=\text { small, } \\
7=\text { large }\end{array}$ & $\begin{array}{l}\text { Extracurricular } \\
\text { activities } \\
1=\text { wide, } \\
7=\text { narrow } \\
\text { selection }\end{array}$ \\
\hline St. John & 3.18 & 3.81 & 3.76 & 3.72 & 4.40 & 3.83 & 4.08 & 3.51 \\
\hline City & 3.28 & 4.19 & 4.93 & 4.47 & 3.95 & 4.15 & 4.10 & 3.85 \\
\hline LIU & 3.73 & 3.71 & 3.79 & 3.89 & 4.21 & 3.96 & 4.00 & 3.69 \\
\hline Baruch & 3.36 & 3.99 & 4.78 & 3.84 & 4.20 & 3.26 & 4.32 & 3.95 \\
\hline Pace & 2.96 & 3.91 & 3.56 & 3.52 & 4.36 & 3.43 & 4.10 & 3.70 \\
\hline Bklyn & 3.07 & 4.25 & 4.95 & 4.44 & 4.10 & 4.52 & 4.12 & 3.89 \\
\hline NYU & 3.25 & 3.21 & 2.76 & 2.51 & 5.13 & 3.17 & 5.07 & 3.29 \\
\hline Queens & 3.90 & 4.14 & 4.52 & 4.39 & 3.92 & 4.45 & 4.33 & 3.89 \\
\hline Wagner & 3.89 & 3.70 & 3.84 & 3.94 & 4.19 & 3.97 & 4.21 & 3.69 \\
\hline JohnJay & 3.66 & 3.87 & 4.34 & 4.22 & 3.91 & 3.61 & 4.08 & 4.09 \\
\hline St.Franc & 3.82 & 4.22 & 4.68 & 4.26 & 4.04 & 4.16 & 3.57 & 3.69 \\
\hline CSI & 4.22 & 4.04 & 4.86 & 4.53 & 3.95 & 4.27 & 4.04 & 3.98 \\
\hline Fordham & 4.21 & 3.69 & 3.77 & 3.73 & 4.32 & 3.80 & 4.44 & 3.70 \\
\hline Hunter & 3.47 & 3.88 & 4.66 & 3.88 & 4.14 & 3.69 & 4.50 & 3.77 \\
\hline Columbia & 3.97 & 2.89 & 2.44 & 2.71 & 5.19 & 3.44 & 4.99 & 3.29 \\
\hline Polytech & 3.59 & 3.78 & 3.51 & 3.44 & 4.13 & 3.44 & 4.06 & 3.99 \\
\hline
\end{tabular}


Table 3: Deviation from midpoint for attribute ratings-1979

\begin{tabular}{|c|c|c|c|c|c|c|c|c|}
\hline & $\begin{array}{l}\text { Transportation } \\
\text { 1=easy, } \\
\text { 7=hard }\end{array}$ & $\begin{array}{l}\text { Campus } \\
\text { facilities } \\
\text { 1=extensive, } \\
7=\text { limited }\end{array}$ & $\begin{array}{l}\text { Cost } \\
\text { 1=high, } \\
7=\text { low }\end{array}$ & $\begin{array}{l}\text { Reputation } \\
\text { 1=high, } \\
\text { 7=low }\end{array}$ & $\begin{array}{l}\begin{array}{l}\text { Course } \\
\text { offerings }\end{array} \\
1=\text { limited, } \\
7=\text { extensive }\end{array}$ & $\begin{array}{l}\text { Educ. } \\
\text { type } \\
1=\text { special, } \\
7=\text { genl. }\end{array}$ & $\begin{array}{l}\text { Student } \\
\text { body } \\
\text { 1=small, } \\
7=\text { large }\end{array}$ & $\begin{array}{l}\text { Extracurricular } \\
\text { activities } \\
1=\text { wide, } \\
7=\text { narrow } \\
\text { selection }\end{array}$ \\
\hline St. John & 0.25 & 0.67 & 0.57 & 0.93 & -1.02 & 0.13 & -0.50 & 1.13 \\
\hline City & 0.47 & -0.27 & -1.85 & -0.57 & -0.73 & -0.77 & -1.50 & 0.15 \\
\hline LIU & -0.67 & 0.02 & 0.00 & -0.15 & -0.80 & -0.27 & -0.63 & 0.32 \\
\hline Baruch & 0.32 & -0.22 & -1.37 & 0.33 & -0.37 & 0.50 & -0.77 & -0.08 \\
\hline Pace & 0.68 & -0.03 & 0.05 & 0.55 & -0.42 & 0.30 & -0.77 & 0.20 \\
\hline Bklyn & 0.95 & 0.05 & -1.72 & -0.37 & -0.57 & -0.98 & -1.42 & 0.18 \\
\hline NYU & 0.83 & 1.03 & 1.38 & 1.55 & -1.65 & 0.50 & -1.77 & 0.88 \\
\hline Queens & -0.28 & 0.30 & -1.45 & 0.25 & -0.60 & -0.67 & -1.37 & 0.35 \\
\hline Wagner & -0.53 & 0.75 & 0.85 & 0.25 & -0.65 & -0.23 & 0.07 & 0.68 \\
\hline JohnJay & -0.05 & -0.73 & -1.18 & -0.07 & 0.34 & 0.92 & 0.23 & -0.38 \\
\hline St.Franc & 0.03 & -0.13 & -0.02 & 0.12 & -0.18 & -0.67 & 0.03 & 0.57 \\
\hline CSI & -0.56 & 0.30 & -1.37 & -0.92 & 0.22 & -0.77 & -0.08 & -0.20 \\
\hline Fordham & -0.25 & 0.52 & 0.48 & 1.08 & -1.00 & 0.38 & -0.60 & 0.27 \\
\hline Hunter & 0.28 & 0.22 & -1.03 & 0.98 & -0.47 & 0.15 & -0.97 & 0.03 \\
\hline Columbia & 0.18 & 1.28 & 1.90 & 2.28 & -1.75 & 0.82 & -1.32 & 0.72 \\
\hline Polytech & -0.20 & 0.17 & 0.63 & 1.33 & -0.37 & 1.70 & 0.00 & -0.20 \\
\hline
\end{tabular}

Table 4: Deviation from midpoint for attribute ratings-2002

\begin{tabular}{|c|c|c|c|c|c|c|c|c|}
\hline & $\begin{array}{l}\text { Transportation } \\
\text { 1=easy, } \\
7=\text { hard }\end{array}$ & $\begin{array}{l}\text { Campus } \\
\text { facilities } \\
1=\text { extensive, } \\
7=\text { limited }\end{array}$ & $\begin{array}{l}\text { Cost } \\
\text { 1=high, } \\
7=\text { low }\end{array}$ & $\begin{array}{l}\text { Reputation } \\
\text { 1=high, } \\
\text { 7=low }\end{array}$ & $\begin{array}{l}\begin{array}{l}\text { Course } \\
\text { offerings } \\
\text { 1=limited, } \\
7=\text { extensive }\end{array}\end{array}$ & $\begin{array}{l}\text { Educ. } \\
\text { type } \\
1=\text { special, } \\
7=\text { genl. }\end{array}$ & $\begin{array}{l}\text { Student } \\
\text { body } \\
1=\text { small, } \\
7=\text { large }\end{array}$ & $\begin{array}{l}\text { Extracurricular } \\
\text { activities } \\
\text { 1=wide, } \\
\text { 7=narrow } \\
\text { selection }\end{array}$ \\
\hline St. John & 0.82 & 0.19 & 0.24 & 0.28 & -0.40 & 0.17 & -0.08 & 0.49 \\
\hline City & 0.72 & -0.19 & -0.93 & -0.47 & 0.05 & -0.15 & -0.10 & 0.15 \\
\hline LIU & 0.27 & 0.29 & 0.21 & 0.11 & -0.21 & 0.04 & 0.00 & 0.31 \\
\hline Baruch & 0.64 & 0.01 & -0.78 & 0.16 & -0.20 & 0.74 & -0.32 & 0.05 \\
\hline Pace & 1.04 & 0.09 & 0.44 & 0.48 & -0.36 & 0.57 & -0.10 & 0.30 \\
\hline Bklyn & 0.93 & -0.25 & -0.95 & -0.44 & -0.10 & -0.52 & -0.12 & 0.11 \\
\hline NYU & 0.75 & 0.79 & 1.24 & 1.49 & -1.13 & 0.83 & -1.07 & 0.71 \\
\hline Queens & 0.10 & -0.14 & -0.52 & -0.39 & 0.08 & -0.45 & -0.33 & 0.11 \\
\hline Wagner & 0.11 & 0.30 & 0.16 & 0.06 & -0.19 & 0.03 & -0.21 & 0.31 \\
\hline JohnJay & 0.34 & 0.13 & -0.34 & -0.22 & 0.09 & 0.39 & -0.08 & -0.09 \\
\hline St.Franc & 0.18 & -0.22 & -0.68 & -0.26 & -0.04 & -0.16 & 0.43 & 0.31 \\
\hline CSI & -0.22 & -0.04 & -0.86 & -0.53 & 0.05 & -0.27 & -0.04 & 0.02 \\
\hline Fordham & -0.21 & 0.31 & 0.23 & 0.27 & -0.32 & 0.20 & -0.44 & 0.30 \\
\hline Hunter & 0.53 & 0.12 & -0.66 & 0.12 & -0.14 & 0.31 & -0.50 & 0.23 \\
\hline Columbia & 0.03 & 1.11 & 1.56 & 1.29 & -1.19 & 0.56 & -0.99 & 0.71 \\
\hline Polytech & 0.41 & 0.22 & 0.49 & 0.56 & -0.13 & 0.56 & -0.06 & 0.01 \\
\hline
\end{tabular}


Table 5: College attribute ratings, change from 1979 to 2002

\begin{tabular}{|c|c|c|c|c|c|c|c|c|}
\hline & $\begin{array}{l}\text { Transportation } \\
\text { 1=easy, } \\
7=\text { hard }\end{array}$ & $\begin{array}{l}\text { Campus } \\
\text { facilities } \\
1=\text { extensive, } \\
7=\text { limited }\end{array}$ & $\begin{array}{l}\text { Cost } \\
\text { 1=high, } \\
7=\text { low }\end{array}$ & $\begin{array}{l}\text { Reputation } \\
\text { 1=high, } \\
7=\text { low }\end{array}$ & $\begin{array}{l}\begin{array}{l}\text { Course } \\
\text { offerings }\end{array} \\
\text { 1=limited, } \\
7=\text { extensive }\end{array}$ & $\begin{array}{l}\text { Educ. } \\
\text { type } \\
1=\text { special, } \\
7=\text { genl. }\end{array}$ & $\begin{array}{l}\text { Student } \\
\text { body } \\
\text { 1=small, } \\
\text { 7=large }\end{array}$ & $\begin{array}{l}\text { Extracurricular } \\
\text { activities } \\
\text { 1=wide, } \\
7=\text { narrow } \\
\text { selection }\end{array}$ \\
\hline St.John & -0.57 & 0.48 & 0.33 & 0.65 & -0.62 & -0.04 & -.42 & 0.64 \\
\hline City & -0.25 & -0.08 & -0.92 & -0.10 & -0.78 & -0.62 & -1.40 & 0.00 \\
\hline LIU & -0.94 & -0.27 & -0.21 & -0.26 & -0.59 & -0.31 & -0.63 & 0.01 \\
\hline Baruch & -0.32 & -0.23 & -0.59 & 0.17 & -0.17 & -0.24 & -0.45 & -0.13 \\
\hline Pace & -0.36 & -0.12 & -0.39 & 0.07 & -0.06 & -0.27 & -0.67 & -0.10 \\
\hline Bklyn & 0.02 & 0.30 & -0.77 & 0.07 & -0.47 & -0.46 & -1.30 & 0.07 \\
\hline NYU & 0.08 & 0.24 & 0.14 & 0.06 & -0.52 & -0.33 & -0.70 & 0.17 \\
\hline Queens & -0.38 & 0.44 & -0.93 & 0.64 & -0.68 & -0.22 & -1.04 & 0.24 \\
\hline Wagner & -0.64 & 0.45 & 0.69 & 0.19 & -0.46 & -0.26 & 0.28 & 0.37 \\
\hline John Jay & -0.39 & -0.86 & -0.84 & 0.15 & 0.25 & 0.53 & 0.31 & -0.29 \\
\hline St.Franc & -0.15 & 0.09 & 0.66 & 0.38 & -0.14 & -0.51 & -0.40 & 0.26 \\
\hline CSI & -0.34 & 0.34 & -0.51 & -0.39 & 0.17 & -0.50 & -0.04 & -0.22 \\
\hline Fordham & -0.04 & 0.21 & 0.25 & 0.83 & -0.68 & 0.18 & -0.16 & -0.03 \\
\hline Hunter & -0.25 & 0.10 & -0.37 & 0.86 & -0.33 & -0.16 & -0.47 & -0.20 \\
\hline Columbia & 0.15 & 0.17 & 0.34 & 0.99 & -0.56 & 0.26 & -0.33 & 0.01 \\
\hline Polytech & -0.61 & -0.05 & 0.14 & 0.77 & -0.24 & 1.14 & 0.06 & -0.21 \\
\hline
\end{tabular}

Table 6: High school seniors' perceptions of academic reputation versus US News \& World Report peer assessment

High School seniors (2002)

Academic reputation*

(1=low quality, $5=$ high quality)

US News \& World Report (2003)

Peer assessment

(1=marginal, $5=$ distinguished $)$

\begin{tabular}{llll}
\hline NYU & 3.92 & Columbia & 4.6 \\
Columbia & 3.79 & NYU & 3.7 \\
Polytechnic & 3.26 & Hunter & 3.3 \\
Pace & 3.20 & Baruch & 3.1 \\
St. John's & 3.06 & Brooklyn & 3.1 \\
Fordham & 3.05 & Fordham & 3.1 \\
Queens & 3.04 & City College & 3.0 \\
Baruch & 2.97 & Queens & 3.0 \\
Hunter & 2.94 & St. Francis & 2.9 \\
LIU & 2.94 & St. John's & 2.8 \\
Wagner & 2.90 & Wagner & 2.7 \\
John Jay & 2.70 & Polytechnic & 2.5 \\
St. Francis & 2.67 & CSI & 2.5 \\
Brooklyn & 2.54 & Pace & 2.4 \\
City College & 2.52 & LIU & 2.3 \\
CSI & 2.48 & & \\
\hline
\end{tabular}

Note: Peer assessment of LIU was listed in US Newes $\&$ World Report under three campuses. LIU CW Post $=2.6$, LIU Brooklyn $=2.3$, LIU Southhampton $=1.9$. Peer assessment of John Jay College was not available.

* Original seven-point scales standardized to a five-point scale, reversed, to facilitate comparison with the US Neres data.

INTERNATIONAL JOURNAL OF EDUCATIONAL ADVANCEMENT. VOL.5 NO.2 107-118 ( ) HENRY STEWART PUBLICATIONS 2005. ISSN 1744-6511. 
survey participants did not perceive them as offering a specialized education.

With the exception of Queens College, the public City University colleges (City College, Baruch, Brooklyn, CSI, and Hunter) have consistently been perceived as low cost. However, the perceptual maps also indicated that they were consistently perceived as low quality. While the perception in 1979 was that these public colleges had large student bodies, this perception appears to have changed over time. Currently, only Columbia and NYU are perceived as having large student bodies. Given the competitive job market, perhaps respondents feel that more students are willing to pay a high price to attend a more prestigious institution. Additionally, over one-third of our 2002 respondents (37\% in 2002 vs $20 \%$ in 1979) indicated that they intended to major in business, thus they may have been more aware of the large student bodies at these institutions with top-rated business programs.

\section{Comparison with US News \& World Report rankings}

High school students' perceptions of colleges do not necessarily match published information about these colleges. For example, many of the colleges in the present survey offer a specialized education in business or engineering, yet respondents did not focus on this. Furthermore, respondents' perceptions regarding the academic reputation of these colleges did not match the more objective measures given by $U S$ Newes \& World Report.

Table 6 compares the ratings given by our sample of high school students on "academic reputation" with US Neres's "peer assessment", described as the assessment made by top academics such as presidents, provosts, and deans of admission at peer institutions. ${ }^{8}$ Tablew 6 is constructed from the data available from the 2003 US Newes \& World Report's online site, which reflects data gathered in 2002. ${ }^{9}$ While our single item construct of academic reputation was not equivalent to US Neres's peer assessment, it did provide a rough means of comparison for the two groups. Because our measure of academic reputation was measured on a seven-point scale, with $1=$ high quality and $7=$ low quality, a simple linear transformation was utilizedto convert our scores to a fivepoint scale to facilitate comparison with the US Neres data. As depicted in Table 6, all of the public universities tended to be perceived by the high school students as being of lower quality, while higher rankings were given for these institutions in US Neres \& World Report.

\section{Recommendations}

High school students' perceptions of New York City colleges have been fairly consistent, although not necessarily accurate. Even on the more mundane attribute of "accessibility by public transportation," perceptions did not match reality. The authors would argue that perhaps with the exception of CSI, located on Staten Island, all of the colleges in this study are easy to reach via public transportation. Some schools may be missing out on attracting students because of the perception that the location is too inconvenient. But the survey indicates that New York City students are willing to travel. In the 2002 survey, 41 percent said they were willing to travel from 30 minutes to an hour, one-way, to attend school while another 23 percent said they would travel for up to one and a half hours one-way.

All but the most prestigious colleges (Columbia and NYU) in our survey appear to suffer from an undistinguished 
profile insofar as they are not perceived as significantly different from average (or each other) on most attributes. In particular, in both 1979 and 2002, St. Francis was not distinguished on any of the eight measured attributes. Yet US Neres o World Report lists St. Francis as a tier 2 comprehensive bachelor's institution for the northeast region.

While the present study provides useful information for college administrators in the New York area, the method could easily be replicated in other areas of the country to gain insights into how the upcoming generation of college students perceives its choices. Additionally, studying high school students offers the opportunity to compare how perceptions change once students graduate from college. This study highlights the need for those colleges that are not in the top tier to develop and implement effective positioning strategies, to differentiate themselves and thus be perceived as more than "average."

\section{References}

1. R. J. Morse and S. M. Flanigan (2001), "How we rank schools: our method and information-packed tables serve families well," US Newes \& World Report, Sept 17, p. 104; G. Garret (2002), "A method explained: our information-packed tables of excellent programs can help you find the right school," US Newes \& World Report, April 15, p. 55.

2. G. Garret (2002), "What the numbers measure." US Neres \& World Report, April 15, p. 72.

3. J. Merritt (2000), “The best b-schools," Business Week, Oct 2, p. 76.

4. B. Cronin (2000), "Rank injustice?" Library Journal, 125, 19, pp. 44-5.

5. E. Segev, A. Raveh, and M. Farjoun M. (1999), "Conceptual maps of the leading MBA programs in the United States: core courses, concentration areas, and the ranking of the school," Strategic Management Journal, 20, pp. 549-65.

6. J. R. Lowry and B. D. Owens (2001), "Developing a positioning strategy for a university," Services Marketing Quarterly, 22, 4, pp. 27-42.

7. L. Hon and B. Brunner (2002), "Measuring public relationships among students and administrators at the University of Florida," Journal of Communication Management, 6, 3, pp. 227-38.

8. < http://www.usnews.com/usnews/edu/college/ rankings/about/index_brief.php>.

9. < http://www.usnews.com/usnews/edu/college/ rankings/rankindex_brief.php>. 


\section{Appendix}

\section{Measures of College Attributes}

Listed below are eight pairs of characteristics which might be considered in selecting a college. Please rate each college on all of the listed qualities, using the seven-point scale as shown.

very easy to reach by

public transportation

$\begin{array}{llllllll}1 & 2 & 3 & 4 & 5 & 6 & 7 & \text { very hard to reach by }\end{array}$

extensive facilities (campus)

public transportation

high cost

$\begin{array}{llllllll}1 & 2 & 3 & 4 & 5 & 6 & 7 & \text { limited facilities (campus) }\end{array}$

high quality academic

$\begin{array}{llllllll}1 & 2 & 3 & 4 & 5 & 6 & 7 & \text { low cost }\end{array}$

reputation

limited number of courses

$\begin{array}{llllllll}1 & 2 & 3 & 4 & 5 & 6 & 7 & \text { low quality academic }\end{array}$

reputation

provides very specialized

education

small student body

wide selection of

extracurricular activities

(clubs \& teams)

$\begin{array}{llllllll}1 & 2 & 3 & 4 & 5 & 6 & 7 & \text { extensive number of courses }\end{array}$

$\begin{array}{llllllll}1 & 2 & 3 & 4 & 5 & 6 & 7 & \text { provides very general }\end{array}$

education

$\begin{array}{llllllll}1 & 2 & 3 & 4 & 5 & 6 & 7 & \text { large student body }\end{array}$

$\begin{array}{llllllll}1 & 2 & 3 & 4 & 5 & 6 & 7 & \text { narrow selection of }\end{array}$

extracurricular activities

(clubs \& teams)

\section{Practitioner's Perspective}

This article's results provide an interesting set of findings relevant for higher education administrators engaged in institutional marketing and student recruitment. Clark suggests that high school seniors' perceptions of colleges and universities remain relatively stable over time. However, it may be disconcerting for practitioners to know that the stability even holds true for misperceptions. The author also demonstrates a tenet of marketing, that for consumers, a correlation exists between cost and perceived institutional quality. Additionally, Clark's piece suggests that individuals with responsibility for crafting institutional images might focus on three areas of practice to increase institutional marketing effectiveness: differentiation, dissemination, and data analyses.

Differentiation: Only NYU and Columbia were perceived as significantly different from the rest of the panel of institutions. This suggests that students have limited abilities to differentiate one institution from another. In the decision-making process, this inability of students to tell what makes an institution a better fit than others may prove problematic for admissions offices. For admissions administrators to successfully recruit students, they must demonstrate to various audiences how their institutions compare to other universities. 
Dissemination: The most effective way for institutions to set themselves apart from competitors is by getting information into the hands of decision makers. Effective dissemination of materials to prospective students and their parents relies upon multiple channels for communication of institutional messages. Additionally, message clarity and consistency will aid in the process of persuading students considering applying or enrolling. This may also prove key to correcting misperceptions about institutional similarities (or differences).

Data analyses: The author also provides a method for admissions professionals to determine changes in institutional perceptions over time. She suggests collecting comparative data to use in evaluating differences between institutions and their competitors. In the article, Clark examines geographic competitors, but the same technique might be applied to other institutions with which one's home institution competes for resources.

Timothy C. Caboni Lecturer in Public Policy and Higher Education, Director, Institutional Advancement Program, Peabody College of Vanderbilt University, Nashville, TN 